\title{
Cardiac Arrhythmias in Patients with COVID-19 Infection - A Single Center Study
}

MD. ABU SALIM, MANZOOR MAHMOOD, DIPAL K ADHIKARY, SAJAL K BANERJEE, MD. HARISUL HOQUE, MA MUQUEET, MD. FAKHRUL I KHALED, WALIDUR RAHMAN, CHAYAN K SINGHA, SUJOY K SAHA, NILUFAR FATEMA, TAREK HASAN, UMMEL KULSUMA, MEHEDI HASSAN, AKM FAZLUR RAHMAN

Department of Cardiology, Bangabandhu Sheikh Mujib Medical University (BSMMU), Shahabag, Dhaka. Address of Correspondence : Dr. Md. Abu Salim, Associate Professor, Department of Cardiology, BSMMU. E-Mail: drsalimsn@hotmail.com

\begin{abstract}
Background: Corona virus disease (COVID-19) has been associated with different cardiac complications including cardiac arrhythmias. Arrhythmias carries a great influence on the outcomes. The pathophysiology of these manifestations remain elusive. This study was aimed to observe the incidence and outcome of cardiac arrhythmia in patients with COVID-19 infection from July 2020 to May 2021.

Method: We conducted an observational study of patients $\geq 18$ years of age with a clinical diagnosis of COVID -19 infection attending Bangabandhu sheikh Mujib Medical University (BSMMU) with and without cardiac arrhythmias from July 2020 to May 2021. Patients were labeled as having arrhythmia if they had sinus bradycardia (heart rate $<40 \mathrm{bpm}), A-V$ block, Atrial fibrillation (AF), atrial flutter (AFL), supraventricular tachycardia (SVT), nonsustained VT, sustained VT, ventricular fibrillation (VF). We excluded those patients presented with Acute Coronary syndrome \& cardiogenic shock. Different clinical data including demographics, comorbidities (like Hypertension, Diabetes mellitus, Chronic kidney disease, IHD), baseline electrocardiographic and echocardiographic findings, antiviral therapy, use of any proarrhythmic drugs.

Results: Data was collected from 2264 patients from BSMMU with COVID-19 infection. Among them 158 patients (6.97\%) had cardiac arrhythmia, 78 (48.91\%) had hypertension, 37 (23.41\%) ischaemic heart disease, 31 (19.62\%) diabetes, 14 (8.88\%) had CKD. Out of 158 patients $53.79 \%$ had sinus bradycardia (heart rate $\leq 40 \mathrm{bpm}) ; 16(10.1 \%)$ had first degree AV block; 14 (8.86 \%) had 2nd or 3rd degree AV block; 27 (17.08\%) had AF; 7 (4.40\%) had AFL; 6 (3.7\%) had SVT; and 3 (1.89\%) of had NSVT. Only one of S. bradycardia patient required temporary pacemaker. 80 (93.75\%) did not require any specific treatment and 4 ( 6.25\%) was ventilated. 14 (8.86\%) had advance AV block, 11 (78.57\%) was discharged, 1 (7.14\%) required permanent pacemaker and $3(21.42 \%)$ needed mechanical ventilation in addition to standard treatment. 40 (25.31\%) had AF/AFL/SVT; all of them were treated with different antiarrhythmic drugs. 40.0\% needed mechanical ventilation and 60.0\% discharged safely. 3 (1.89\%) had NSVT or VT, 2 (66.67\%) of them needed mechanical ventilation and 1 (33.33\%) was discharged safely. 87.32\% patient was discharged safely and $16.4 \%$ was ventilated. Among 26 ventilated patients 15 (57.69\%) was expired. Out of them 11 (68.75\% of 16 patient) had atrial tachyarrhythmia, 2 (100\% of 2 patient) had ventricular tachyarrhythmia and 2 (16.67\% of 12 patient) had bradyarrhythmia.
\end{abstract}

Conclusion: COVID-19 infection made a profound negative effect on the lives of millions of people across the world. More than 3 millions of people already died. Lots of patients of COVID-19 infection developed cardiac arrhythmia and was associated with high morbidity and mortality. Appropriate monitoring by ECG with accurate and early identification of arrhythmia is important for better management and outcome.

University Heart Journal 2022; 18(1): 61-64

Introduction:

Since December 2019 Corona virus infection is the burning health problem in the globe. In December 2019 a novel corona virus was identified as the cause of a cluster of pneumonia cases in Wuhan, China. From then it rapidly spread and became global pandemic. Till today coronavirus disease (COVID-19) has affected more than 150 million people leading to over 3 million deaths worldwide. ${ }^{1}$ It is well known that COVID-19 can affect nearly every organ system of human body. In 
cardiovascular system it can cause different complications, among them cardiac arrhythmia is an important complication. Although the underlying mechanism has remained elusive, different studies and surveys around the world have reported different electrophysiological issues associated with the disease, specifically atrioventricular block, atrial fibrillation (AF), polymorphic ventricular tachycardia. ${ }^{2-5}$ Coexisting electrolyte imbalance, hypoxia and use of proarrhythmic medications (e.g. hydroxychloroquine, azithromycin) makes it difficult to understand the direct contribution of COVID-19 on cardiac arrhythmia.

A significant increase (58\%) of out of hospital cardiac arrest during the first 40 days of COVID-19 pandemic in the Lombardy region of Italy raised concerns regarding the risk of arrhythmia associated with COVID-19 infection. ${ }^{6}$ In the United States a single center study of 700 patients demonstrated that the patients admitted in ICU were associated with a 10 fold increase of arrhythmia risk. ${ }^{7}$ Most of the arrhythmias were sinus bradycardia, incidental atrial fibrillation and non-sustained ventricular tachycardia (NSVT) and most of which were not associated with mortality. ${ }^{6}$

In Bangladesh for last 15-month coronavirus disease (COVID-19) has affected more than 750 thousand people leading to over 12,100 deaths. ${ }^{8}$ In Bangabandhu Sheikh Mujib Medical university (BSMMU) we have observed varieties of conductive system disorders and cardiac arrhythmia in COVID-19 infected patients.

\section{Method:}

We conducted an observational study of patients $\geq 18$ years of age with a clinical diagnosis of COVID -19 infection (SARS-CoV-2) confirmed by nasopharyngeal RT-PCR testing attending the OPD and admitted in hospital with and without incident cardiac arrhythmias from July 2020 to May 2021. Patients were labeled as having arrhythmia from the 12 lead electrocardiography (ECG) if they had marked sinus bradycardia (heart rate $<40 \mathrm{bpm}$ ), atrioventricular block, Atrial fibrillation (AF), atrial flutter (AFL), supraventricular tachycardia (SVT), nonsustained VT, sustained monomorphic or polymorphic VT, ventricular fibrillation (VF). We excluded those patients presented with Acute Coronary syndrome \& cardiogenic shock.

Different Clinical data including demographics, comorbidities (like Hypertension, Diabetes mellitus, Chronic kidney disease, IHD, malignancy), baseline electrocardiographic and echocardiographic findings, antiviral therapy and use of any proarrhythmic drugs (hydroxychloroquine, azithromycin).

\section{Results:}

Total 2264 patients with COVID-19 infection were screened, among them 1712 patient at out patients department (OPD) and 552 hospitalized patients. Among them 158 patients $(6.97 \%)$ had cardiac arrhythmia as a result of COVID-19 infection.

Mean age of the total covid-19 infected patients with or without arrhythmias was $56.18 \pm 15.0$ years, and $63 \%$ were men and $37 \%$ were female. Table: 1 shows that Out of 2264 patients $48.10 \%$ was hypertensive ( $M=48.91 \%$ and $\mathrm{F}=46.71 \%) ; 31.22 \%$ was diabetic ( $\mathrm{M}=33.07 \%$, $\mathrm{F}=27.95 \%) ; 8.25 \%$ had ischaemic heart disease $(\mathrm{M}=8.68 \%, \mathrm{~F}=7.52 \%) ; 3.84 \%$ had chronic kidney disease.

Among 158 patients ( 6.97\%) of arrhythmia, 94 (59.49\%) patient was male and $64(40.50 \%)$ was female; 78 (49.36\%) was hypertensive; 31 (19.62\%) was diabetic; $37(23.41 \%)$ had history of ischaemic heart disease; 14 (8.88\%) was known CKD.

Majority of the COVID-19 patients who developed cardiac arrhythmia had no previous history of arrhythmia. All of them were followed-up for 30 days from the RT-PCR for COVID-19 was positive. Table. 3 shows, 132 (87.32\%) patient was discharged safely and 26 (16.4\%) was ventilated. Among 85 (53.79\%) patients who had Sinus Bradycardia (heart rate $\mathrm{d}+40 \mathrm{bpm}$ ) only one of them required temporary pacemaker for 6 days and discharged safely, rest of the 84 patients did not required any medication to increase heart rate. $16(10.1 \%)$ had $1^{0} \mathrm{AV}$ block, $15(93.75 \%)$ did not require any specific treatment for this, $1(6.25 \%)$ of them was ventilated for severe hypoxia. $14(8.86 \%)$ had advanced AV block, of them 11 (78.57\%) was discharged safely, 1 (7.14\%) needed permanent pacemaker and $3(21.42 \%)$ needed mechanical ventilation. Out of 158 patients $40(25.31 \%)$ had AF/AFL/ SVT, most of them $27(67.5 \%)$ was treated with beta blocker (Metoprolol or Bisoprolol), 4 (10\%) was treated with Amiodarone, 6 (15.0\%) with sotalol, 3 (7.5\%) with $\mathrm{Ca}++$ channel blocker (verapamil or diltiazem), $16(40.0 \%)$ needed mechanical ventilation and 24 (60.\%) were discharged safely. Out of $3(1.89 \%)$ patients who had NSVT or VT, $2(66.67 \%)$ of them needed mechanical ventilation and $1(33.33 \%)$ was discharged safely. Among the 26 ventilated patient $15(57.69 \%)$ were expired. 11 (68.75\% of 16 patient) had atrial tachyarrhythmia, 2 (100\% of 2 patient) had ventricular tachyarrhythmia and 2 (16.67\% of 12 patient) had bradyarrhythmia. 
Table-I

Clinical Characteristics of Patients with COVID-19 with and without Arrhythmia

\begin{tabular}{lcccc}
\hline Medical Comorbidities & \multicolumn{2}{c}{ Total number of patient } & \multicolumn{2}{c}{$\begin{array}{c}\text { Number of patients with cardiac } \\
\text { arrhythmia n=158 } \\
\end{array}$} \\
& Male=1427 & Female=837 & Male=94 & Female=64 \\
\hline Hypertension & $698(48.91 \%)$ & $391(46.71 \%)$ & $56(59.57 \%)$ & $22(34.37 \%)$ \\
Diabetes Mellitus & $472(33.07 \%)$ & $235(27.95 \%)$ & $18(19.14 \%)$ & $13(20.31 \%)$ \\
Ischaemic Heart Disease & $124(8.68 \%)$ & $63(7.52 \%)$ & $29(30.85 \%)$ & $8(12.5 \%)$ \\
Chronic Kidney disease & $56(3.92 \%)$ & $31(3.7 \%)$ & $9(9.57 \%)$ & $5(7.81 \%)$ \\
Others (Malignancy, Stroke) & $18(1.26 \%)$ & $7(0.83 \%)$ & $8(8.51 \%)$ & $7(10.93 \%)$ \\
\hline
\end{tabular}

Table-II

Types of Cardiac Arrhythmias in patient with COVID-19 infection

\begin{tabular}{lcc}
\hline Type of arrhythmia & \multicolumn{2}{c}{ Total number = 158 (\%) } \\
\cline { 2 - 3 } & Male=94(59.49\%) & Female =64 (40.5) \\
\hline S. Bradycardia $=85(53.79 \%)$ & $51(60 \%)$ & $34(40 \%)$ \\
$1^{0}$ AV block $=16(10.1 \%)$ & $9(56.25 \%)$ & $7(43.75 \%)$ \\
Advance AV block $=14(8.86 \%)$ & $9(64.28 \%)$ & $5(35.71 \%)$ \\
Atrial fibrillation $(\mathrm{AF})=27(17.08 \%)$ & $18(66.66 \%)$ & $9(33.33 \%)$ \\
Atrial flutter $(\mathrm{AFL})=7(4.4 \%)$ & $5(71.42 \%)$ & $2(28.57 \%)$ \\
Supraventricular tachycardia $=6(3.7 \%)$ & $4(66.67 \%)$ & $2(33.33 \%)$ \\
Ventricular tachycardia $(\mathrm{VT})=3(1.89 \%)$ & $3(100 \%)$ & - \\
\hline
\end{tabular}

Table-III

Arrhythmia management and outcome of patients with COVID-19 infection with Arrhythmia

\begin{tabular}{|c|c|c|c|c|c|c|}
\hline & $\begin{array}{c}\text { All; } \\
\mathrm{N}=158\end{array}$ & $\begin{array}{c}\text { S. Bradycardia } \\
\mathrm{n}=85(53.79 \%)\end{array}$ & $\begin{array}{c}1^{0} \mathrm{AV} \\
\text { block } \\
\mathrm{n}=16 \\
(10.1 \%) \\
\end{array}$ & $\begin{array}{c}\text { Advance } \\
\text { AV block } \\
n=14 \\
(8.86 \%) \\
\end{array}$ & $\begin{array}{c}\text { AF, AFL, } \\
\text { SVT n=40 } \\
(25.31 \%)\end{array}$ & $\begin{array}{c}\text { NSVT / VT } \\
n=3 \\
(1.89 \%)\end{array}$ \\
\hline \multicolumn{7}{|l|}{ Treatment } \\
\hline Â-blocker & & - & - & - & 27 & 3 \\
\hline Amiodarone & & - & - & - & 4 & 2 \\
\hline Sotalol & & - & - & - & 6 & - \\
\hline $\mathrm{Ca}^{++}$channel blocker & & - & - & - & 3 & - \\
\hline Other AAD & & - & - & - & - & - \\
\hline Pacemaker & & 1 & - & 1 & - & - \\
\hline Outcome & & & & & & \\
\hline $\begin{array}{l}\text { Survival to } \\
\text { discharge }\end{array}$ & $\begin{array}{c}132 \\
(87.32 \%)\end{array}$ & 81 & 15 & 11 & 24 & 1 \\
\hline Ventilated & $26(16.4 \%)$ & 4 & 1 & 3 & 16 & 2 \\
\hline Death & $15(9.49 \%)$ & 1 & - & 1 & 11 & 2 \\
\hline
\end{tabular}

Majority of the COVID-19 patients who developed cardiac arrhythmia had no previous history of arrhythmia. All of them were followed-up for 30 days from the RT-PCR for COVID-19 was positive. Table III shows, $132(87.32 \%)$ patient was discharged safely and 26 (16.4\%) was ventilated. Among 85 (53.79\%) patients who had Sinus Bradycardia (heart rate $\leq 40 \mathrm{bpm}$ ) only one of them required temporary pacemaker for 6 days and discharged safely, rest of the 84 patients did not required any medication to increase heart rate. $16(10.1 \%)$ had $1^{0} \mathrm{AV}$ 
block, $15(93.75 \%)$ did not require any specific treatment for this, $1(6.25 \%)$ of them was ventilated for severe hypoxia. 14 (8.86\%) had advanced AV block, of them 11 (78.57\%) was discharged safely, $1(7.14 \%)$ needed permanent pacemaker and $3(21.42 \%)$ needed mechanical ventilation. Out of 158 patients $40(25.31 \%)$ had AF/AFL/ SVT, most of them $27(67.5 \%)$ was treated with beta blocker (Metoprolol or Bisoprolol), 4 (10\% ) was treated with Amiodarone, 6 (15.0\%) with sotalol, 3 (7.5\%) with $\mathrm{Ca}++$ channel blocker (verapamil or diltiazem), $16(40.0 \%)$ needed mechanical ventilation and 24 (60.\%) were discharged safely. Out of $3(1.89 \%)$ patients who had NSVT or VT, $2(66.67 \%)$ of them needed mechanical ventilation and 1(33.33\%) was discharged safely. Among the 26 ventilated patient 15 (57.69\%) were expired. 11 (68.75\% of 16 patient) had atrial tachyarrhythmia, 2 (100\% of 2 patient) had ventricular tachyarrhythmia and 2 (16.67\% of 12 patient) had bradyarrhythmia.

\section{Discussion:}

In a large number of patients with COVID-19 infection, arrhythmias were common and associated with high morbidity and mortality. Sinus bradycardia (heart rate d" $40 \mathrm{bpm}$ ) was most common, occurring in $53.79 \%$ of patients with arrhythmia, next to that was atrial arrhythmias (25.31\% Patients with cardiac arrhythmias). Cardiac arrhythmias had a high burden of medical comorbidities, although most of them did not have a prior history of arrhythmia. Al most half (49.36\%) of them was hypertensive, $23.41 \%$ of them has history of ischaemic heart disease, $19.62 \%$ was diabetic and $8.88 \%$ was known CKD. Regarding the management, most of the patients with Brady-arrhythmias, were managed by observation and withdrawal of any offending heart rate limiting drugs and correction of any electrolytes derangements. Among tachyarrhythmias $69.76 \%$ were treated with beta blockers, $13.95 \%$ were treated with amiodarone, $13.95 \%$ were treated with sotalol and $6.9 \%$ were treated with different $\mathrm{Ca}^{++}$channel blockers.

About the outcome of treatment of the patients of COVID19 infection with cardiac arrhythmias, most of the patients (87.32\%) was discharged safely and $16.4 \%$ was needed mechanical ventilation support. Patient with tachyarrhythmias had poor outcome, $40 \%$ of atrial tachyarrhythmia patients needed mechanical ventilation and $66 \%$ ( 2 out of 3 patient) of ventricular tachyarrhythmia was ventilated. Bradyarrhythmia patients had a relatively better outcome, $93.04 \%$ (107 out of 115 patients) was discharged safely, $1.73 \%$ ( 2 out of 115 patients) needed pacemaker support and $6.9 \%$ (8 out of 115 patients) needed mechanical ventilation.

\section{Conclusions:}

Novel corona virus (COVID-19) infection made a profound negative effect on the lives of millions of people across the world. More than 3 millions of people already died due to this disease and billions of people have suffered both physically and financially. Lots of patients of COVID19 infection developed cardiac arrhythmia and was associated with high morbidity and mortality and highlights the need for electrophysiologists to be involved in COVID-19 patients care. Future investigations regarding the mechanisms of the cardiac complications of COVID-19 (SARS-CoV-2) infection may aid in our understanding of arrhythmias.

\section{References:}

1. https://www.worldometers.info/coronavirus/ accessed online on 14 may 2021.

2. Kochav SM, Coromilas E, Nalbandian A, Ranard LS, Gupta A, Chung MK, Gopinathannair R, Biviano AB, Garan H, Wan EY. Cardiac arrhythmias in COVID-19 infection. Circ Arrhythm Electrophysiol. 2020;13:e008719. doi: 10.1161/CIRCEP. 120.008719 .

3. Wang D, Hu B, Hu C, Zhu F, Liu X, Zhang J, Wang B, Xiang H, Cheng Z, Xiong Y, et al. Clinical Characteristics of 138 Hospitalized Patients With 2019 Novel Coronavirus-Infected Pneumonia in Wuhan, China. JAMA. 2020;323:1061-69. doi: 10.1001/jama.2020.1585.

4. Elias P, Poterucha TJ, Jain SS, Sayer G, Raikhelkar J, Fried J, Clerkin K, Griffin J, DeFilippis EM, Gupta A, et al. The prognostic value of electrocardiogram at presentation to emergency department in patients with COVID-19. Mayo Clin Proc. 2020;95:2099-109. doi: 10.1016/j.mayocp. 2020.07.028.

5. Gopinathannair R, Merchant FM, Lakkireddy DR, Etheridge SP, Feigofsky S, Han JK, Kabra R, Natale A, Poe S, Saha SA, et al. COVID19 and cardiac arrhythmias: a global perspective on arrhythmia characteristics and management strategies. J Interv Card Electrophysiol. 2020;59:329-36. doi: 10.1007/s10840-02000789-9.

6. Baldi E, Sechi GM, Mare C, Canevari F, Brancaglione A, Primi R, Klersy C, Palo A, Contri E, Ronchi V, et al; Lombardia CARe Researchers. Out-ofHospital Cardiac Arrest during the Covid-19 Outbreak in Italy. N Engl J Med. 2020;383:496-98. doi: 10.1056/ NEJMc2010418.

7. Bhatla A, Mayer MM, Adusumalli S, Hyman MC, Oh E, Tierney A, Moss J, Chahal AA, Anesi G, Denduluri S, et al. COVID-19 and cardiac arrhythmias. Heart Rhythm. 2020;17:1439-44. doi: 10.1016/j.hrthm.2020. 06.016

8. https://www. worldometers. info/ coronavirus/ co untry/ bangladesh/ accessed online on 14 may 2021. 